\title{
Editorial
}

\section{Prion Inactivation and Medical Instrument Reprocessing: Challenges Facing Healthcare Facilities}

\author{
Lynne M. Sehulster, PhD, M(ASCP)
}

The major transmissible spongiform encephalopathies (TSEs) of humans include Creutzfeldt-Jakob disease (CJD), kuru, fatal familial insomnia, GerstmannSträussler-Scheinker syndrome, and, within the past 10 years, variant CJD (vCJD). ${ }^{1-3}$ The pathology of these neurodegenerative diseases of the central nervous system is associated with the presence of pathologic prions, an abnormal conformation of a normal cellular protein. ${ }^{4}$ Fomites and tissues contaminated with prions, such as dura mater and corneas, can transmit disease to humans. ${ }^{5}$ At least six cases of CJD have been attributed to the use of neurosurgical instruments and depth electrodes contaminated with prion-containing neural tissues. ${ }^{6}$ These cases of device-associated transmission highlight the challenges and data gaps that confront healthcare staff responsible for disinfection and sterilization of hospital equipment. The work described by Yan et al. ${ }^{7}$ in the lead article of this issue of Infection Control and Hospital Epidemiology represents an attempt to address the gap in our knowledge regarding prion decontamination and low-temperature sterilization methods.

Pioneering research conducted at the National Institutes of Health in the early to mid-1980s provided evidence that prion proteins demonstrated significant resistance to conventional sterilization and disinfection methods.$^{8.9}$ A recent review by Taylor ${ }^{10}$ reveals the complexity of prion inactivation. Most of the reported studies focused on prion inactivation in tissues. A remaining question is, how applicable is this research to surgical instrument reprocessing? Can laboratory-based inactivation methods be applied to central sterilization department operations? Three major conclusions can follow from review of the literature
First, prion inactivation research was not designed to assess instrument reprocessing strategies until only recently. Most prior research had focused on inactivating prions present in small amounts of whole brain tissue or neural tissue homogenates. It is only the more recent work that has studied prion inactivation on surfaces. If this contaminated neural tissue is present on surfaces (eg, neurosurgical instruments), a high prion burden embedded in organic material would pose a significant challenge to any inactivation method. Cleaning the surface to remove gross tissue should reduce the prion burden. ${ }^{11,12}$ Recent research showing that prions bind tightly to steel underscores this importance of cleaning to remove tissue. ${ }^{13,14}$ If prion-containing material is dried or heat-fixed onto surfaces, unusually thermostable subpopulations of prions remain even after autoclaving; animal assays have shown that infectivity persists. ${ }^{15}$ These studies indicate that instruments contaminated with prion material should not be allowed to dry prior to a thorough cleaning or decontamination process.

Second, most research has used experimental designs that do not resemble contemporary commercial or routine practices of medical instrument reprocessing in healthcare. Prion inactivation data suggest that a more rigorous approach is needed. Research in laboratories around the world has shown that adding a chemical hydrolysis step (eg, use of sodium hydroxide $[\mathrm{NaOH}]$ ) into prion inactivation strategies is a relatively effective means of reducing prion titers in neural tissue as demonstrated in animal assay experiments. ${ }^{16}$ Combining the use of $\mathrm{NaOH}$ as an instrument immersion step with autoclaving in a gravitydisplacement sterilizer was deemed a particularly effective strategy for prion inactivation. ${ }^{17}$ This approach, however,

Dr. Sehulster is from the Epidemiology and Laboratory Branch, Division of Healthcare Quality Promotion, National Center for Infectious Diseases, Centers for Disease Control and Prevention, Atlanta, Georgia.

Address reprint requests to Lynne Sehulster, $P h D, M(A S C P)$, Mailstop A-35, DHQP, Centers for Disease Control and Prevention, 1600 Clifton Road NE, Atlanta, GA 30333

Use of trade names is intended for identification purposes only and does not represent endorsement by either the CDC, the United States Public Health Service, or the Department of Health and Human Services. 
can be hazardous to both the autoclave equipment and the operators if $\mathrm{NaOH}$ is not properly contained within an appropriate vessel. ${ }^{17,18}$ Alternative decontamination strategies for prion-contaminated instruments employ an initial immersion into sodium hypochlorite solutions of 20,000 ppm or greater of total available chlorine. ${ }^{10}$

Finally, the materials used in prion inactivation experiments often differ from surgical instruments used in patient care. Medical instruments and devices range from simple steel items to complex tools, some made with heat-sensitive materials. Some of these complex instruments have power motors, electronics, optics, and lumens that present a challenge to conventional reprocessing. Delicate instruments can be damaged when exposed to $\mathrm{NaOH}$, sodium hypochlorite, or autoclaving. In contrast, small, simple, solid carriers (eg, steel wires) with smooth surfaces are often used in many inactivation experiments to facilitate intracerebral implantation into assay animals. This experimental design necessity does not address the challenge of reprocessing a complex medical instrument with uneven surfaces, hinges, or differing materials. If prion inactivation problems are encountered when decontaminating a simple steel wire, there will likely be significant challenges encountered when decontaminating a complex instrument.

There is a paucity of information regarding prion decontamination methods using modern low-temperature inactivation processes. While steam sterilization remains the mainstay in healthcare facilities, low-temperature sterilization processes are vital for the terminal reprocessing of delicate and heat-sensitive instruments, the performance of which would be compromised if they were exposed to either single or repeated cycles of steam under pressure. The findings of Yan et al. ${ }^{7}$ in this issue of Infection Control and Hospital Epidemiology provide important new information. The experimental design incorporates a cleaning step using either of two cleaning agents (ie, an enzymatic cleaner and an alkaline cleaner). However, neither of these chemicals is identified specifically beyond a generic description of the active ingredient. Comparisons are made among untreated controls and a variety of decontamination strategies to evaluate residual prion activity on steel wires exposed to homogenates of central nervous system tissue. Low-temperature decontamination strategies included use of $59 \%$ hydrogen peroxide, $0.35 \%$ peracetic acid (NU-Cidex, Johnson \& Johnson Medical, Ltd., High Wycombe, United Kingdom), $0.55 \%$ ortho-phthalaldehyde (Cidex OPA, Advanced Sterilization Products, Irvine, CA), and hydrogen peroxide gas plasma (Sterrad, Advanced Sterilization Products). Two of the agents (hydrogen peroxide and peracetic acid) were evaluated as simple immersion processes without the use of an initial cleaning. The processes using the hydrogen peroxide gas plasma sterilizer and orthophthalaldehyde were examined with and without cleaning. Comparisons were made with a current prion inactivation strategy involving $\mathrm{NaOH}$ and steam autoclaving at $134^{\circ} \mathrm{C}$ for 18 minutes. However, the description of this approach suggests that immersion in $\mathrm{NaOH}$ was a separate step per- formed before subjecting the wires to autoclaving, rather than using the more familiar strategy of autoclaving items while they are immersed in $\mathrm{NaOH}$ directly. Currently, the latter approach is the preferred prion inactivation method from World Health Organization (WHO) and Centers for Disease Control and Prevention recommendations for heatstable instruments if discarding and incinerating the contaminated instruments is not an option..$^{19,20}$ Estimates of residual infectivity were based on the numbers of surviving animals and the time interval between exposure and the onset of symptoms in affected animals.

Readers should interpret the results with cautious enthusiasm and consider this as the first installment of continuing research to evaluate low-temperature sterilizing processes and prion inactivation. It is not clear why the authors used a model of hydrogen peroxide gas plasma sterilizer (Sterrad 100S GMP) not available to healthcare facilities to sterilize soft steel wires in preparation for the experiment, whereas the sterilizer marketed to healthcare facilities (Sterrad 100S) was used during the experiment phase. More importantly, the results presented in the article suggest that the use of an alkaline detergent wash at $70^{\circ} \mathrm{C}$ followed by a cycle in the Sterrad $100 \mathrm{~S}$ sterilizer using four injections of hydrogen peroxide gas plasma has sufficient potency to inactivate prions. The authors acknowledge that special software modifications were made to the Sterrad 100 S sterilizer to accomplish the four injections of gas plasma during the cycle. The modifications were made specifically for research; the commercially available Sterrad $100 \mathrm{~S}$ sterilizer has a fixed two-injection cycle. Further studies may need to examine the effects of running two consecutive, two-injection cycles on prion inactivation.

Research to develop effective yet "instrument-friendly" prion inactivation strategies that reflect the daily operations and capabilities of the instrument reprocessing departments of healthcare facilities cannot come soon enough. Four issues arise in considering the infection control challenges of prion inactivation facing hospitals. First, current prion inactivation strategies invariably damage surgical instruments, the replacement of which can represent enormous cost. The current worldwide standard for both TSE patient management and instrument reprocessing strategies is the 1999 WHO consensus statement. ${ }^{19}$ Its guidance on instruments is conservative; the method with the highest level of safety involves disposing of contaminated instruments via incineration. This approach encourages use of single-use, disposable instruments in place of durable instruments for surgeries in which there is potential for significant prion contamination. This is not always the best option, especially if the single-use instrument is inferior to the durable instrument, or leads to worse patient outcomes. Such was the case during the recent period in the United Kingdom when single-use instruments were recommended for non-emergency tonsillectomies and adenoidectomies in attempts to manage theoretical risks of exposure to vCID prions. ${ }^{21,22}$ Rates for postoperative hemorrhaging increased dramatically when single-use instru- 
ments were used, ranging from $7.8 \%$ to $12 \%$, compared with approximately $1 \%$ when durable instruments were used. The recommendation has since been rescinded by United Kingdom health authorities.

WHO recommendations for reusable instrument reprocessing involve combinations of immersion in $\mathrm{NaOH}$ and steam autoclaving using higher temperatures and longer cycles as compared with conventional cycle settings. ${ }^{19}$ Other WHO options describe exposing instruments to high concentrations of sodium hypochlorite, followed by either rinsing or immersing the instruments in water and autoclaving the instruments at the high temperature-longer cycle settings. ${ }^{19}$ Both methods will damage or discolor instruments. Development of the WHO recommendations involved various scientists with expertise in prion inactivation research. However, additional input from medical instrument and sterilizer manufacturers would have provided greater consideration of issues affecting practical implementation. Discussions at the U.S. Food and Drug Administration Transmissible Spongiform Encephalopathy Advisory Panel meeting on July 17 and 18, 2003, highlighted the importance of these practical perspectives and their contribution to studies on contaminated instrument reprocessing. ${ }^{23}$ Additionally, the panel called for a future meeting of all interested stakeholders on this issue (eg, neurosurgeons, laboratory researchers, risk managers, infection control professionals, and manufacturers) to assess the "state of the art." This is an important direction to better understand the nature of prion inactivation and prion transmission via fomites.

A second challenge is that hospitals need to ensure that perioperative diagnoses of TSE diseases are made quickly so that instruments can be managed appropriately and situations requiring instrument recall and patient notification can be avoided. Healthcare facilities need to establish policies and procedures to govern instrument management during TSE patient care. ${ }^{24}$ If there is clinical suspicion that a surgical case poses a high risk of prion contamination for instruments and a prion-specific reprocessing strategy is elected, the instruments should be kept moist until such time when tissue removal and prion decontamination can be initiated. When neurosurgery is performed for diagnosis, some institutions may "quarantine" the instruments until the diagnosis is returned, thereby limiting the damaging effects of prion-specific reprocessing to only those instruments used on CJD-diagnosed patients. This approach is best suited for those instances when a diagnosis can be made as rapidly as possible. Other institutions may choose to proceed with prion-specific reprocessing whenever there is doubt about the status of the surgical case. Some institutions have run cost-effectiveness studies on their TSE patient case load and determined that discarding the instruments is the best strategy for their facility.

A third challenge is that uncertainties exist regarding extraneural concentrations of prions and the potential for iatrogenic transmission. The WHO guidance assigns various tissues and organ systems to risk categories ranging from "high" to "no infectivity." 19 The central nervous sys- tem tissues clearly represent the highest risk for prion contamination, and in the United States the focus has been primarily on neurosurgical instruments. Recent evidence of pathologic prions in spleen and muscle tissues of a small sample of sporadic CJD patients advances our understanding of the molecular and physiologic basis of the disease, but the impact of this information on risk management strategies for surgical instruments is unknown at this time. ${ }^{25}$ Our colleagues in Europe face an even greater challenge, given the knowledge that lymphoid tissues (eg, tonsils and spleen) of vCJD patients harbor significant concentrations of prions, albeit at levels clearly less than those in central nervous system tissues. ${ }^{26}$ Iatrogenic transmission of vCJD resulting from lymphoid tissue contamination of surgical instruments has not been described, but the potential for transmission is such that European healthcare professionals are looking for strategies to reduce potential transmission from instrument contact with lymphoid tissue. In addition, the United Kingdom's National Creutzfeldt-Jakob Disease (CJD) Surveillance Unit has announced that it is making endoscopes available for use only on diagnosed patients with either classic sporadic CJD or vCJD, thereby helping hospitals to limit potential contamination of their endoscope inventory. ${ }^{27}$

A fourth challenge is that uncertainties exist regarding the potential for transmission from patients who may be incubating a TSE but who are asymptomatic at the time of surgery. There is renewed interest in the possible role of the "preclinical" TSE patient with respect to prion contamination of medical instruments, due to a recent report of potential transmission of VCJD in the United Kingdom via blood transfusion from a donor diagnosed after donation. ${ }^{28}$ Investigations using experimentally infected animals have documented the presence and accumulation of abnormal prions in at-risk tissues while the animals remain asymptomatic. ${ }^{29}$ This information would suggest that a "carrier" state exists for TSEs in animals. It is possible that this could very well be the case for humans. However, this currently remains an intriguing speculation, given that there are no easy, reliable, and approved commercial methods to identify a CJD infection in patients without symptoms or a history of risk factors.

There is excitement among prion inactivation researchers as new knowledge becomes available, and healthcare facility professionals are eager for guidance that eliminates uncertainties in risk assessment while ensuring patient safety and effective management of resources. The findings presented by Yan et al. ${ }^{7}$ move us further toward understanding and developing improved, practical methods for prion inactivation. There will come a time in the near future when prion inactivation research will result in methods that can be subjected to process validation that can withstand regulatory agency scrutiny. ${ }^{23}$ To that end, it is important that researchers ensure that their methods yield measurable, objective data $\left(\mathrm{eg}, \log _{10}\right.$ reductions in prion titers) whenever possible. Until that time, the issue of prion inactivation is likely to remain a complex and evolving subject. 


\section{REFERENCES}

1. Collins SJ, Lawson VA, Masters CL. Transmissible spongiform encephalopathies. Lancet 2004;363:51-61.

2. Will RG, Ironside JW, Zeidler MK, et al. A new variant of CreutzfeldtJakob disease in the UK. Lancet 1996;347:921-925.

3. Beisel CE, Morens DM. Variant Creutzfeldt-Jakob disease and the acquired and transmissible spongiform encephalopathies. Clin Infect Dis 2004;38:697-704

4. Pruisner SB. Novel proteinaceous infectious particles cause scrapie. Science 1982;216:136-144.

5. Will RG. Acquired prion disease: iatrogenic CJD, variant CJD, kuru. British Medical Bulletin 2003;66:255-265.

6. Bernoulli C, Sigfried J, Baumgarten G, et al. Danger of accidental person to person transmission of Creutzfeldt-Jakob disease by surgery. Lancet 1977;1:478-479.

7. Yan Z, Stitz L, Heeg P, Pfaff E, Roth K. Infectivity of prion protein bound to stainless steel wires: a model for testing decontamination procedures for transmissible spongiform encephalopathies. Infect Control Hosp Epidemiol 2004;25:280-283.

8. Asher DM, Pomeroy KL, Murphy L, Rohwer, RG, Gibbs CJ, Gajdusek DC. Practical inactivation of scrapie agent on surfaces. Presented at the IXth International Congress of Infectious and Parasitic Diseases; July 2026, 1986; Munich, Germany.

9. Asher DM, Pomeroy KL, Murphy L, Gibbs CJ, Gajdusek DC. Attempts to disinfect surfaces contaminated with etiological agents of the spongiform encephalopathies. Presented at the VIIth International Congress of Virology; August 9-14, 1987; Edmonton, Alberta, Canada.

10. Taylor DM. Inactivation of transmissible degenerative encephalopathy agents: a review. Vet $J 2000 ; 159: 10-17$.

11. Favero MS. Current issues in hospital hygiene and sterilization technology. Journal of Infection Prevention Asia Pacific 1998;1:8-10.

12. Favero MS, Bond WW. Chemical disinfection of medical and surgical instruments. In: Block SS, ed. Disinfection, Sterilization, and Preservation, ed. 5. Philadelphia: Lippincott Williams \& Wilkins; 2001:881-917

13. Zobeley E, Flechsig E, Cozzio A, Enari M, Weissmann C. Infectivity of scrapie prions bound to a stainless steel surface. Mol Med 1999:5:240 243.

14. Flechsig E, Hegyi I, Enari M, Schwarz P, Collinge J, Weissmann C Transmission of scrapie by steel-surface-bound prions. Mol Med 2001;7:679-684.

15. Taylor DM, Fernie K, McConnell I, Steele PJ. Observations on thermostable subpopulations of the unconventional agents that cause transmissible degenerative encephalopathies. Vet Microbiol 1998;
64:33-38.

16. Ernst DR, Race RE. Comparative analysis of scrapie agent inactivation methods. I Virol Methods 1993;41:193-202.

17. Taylor DM, Fernie K, McConnell I. Inactivation of the 22A strain of scrapie agent by autoclaving in sodium hydroxide. Vet Microbiol 1997; 58:87-93.

18. Brown SA, Merritt $\mathrm{K}$. Use of containment pans and lids for autoclaving caustic solutions. Am I Infect Control 2003;31:257-260.

19. World Health Organization. WHO infection control guideline for transmissible spongiform encephalopathies: report of a WHO consultation. Geneva, Switzerland: World Health Organization; 1999. Available at www.who.int/emc-documents/tse/whocdscsraph2003c.html.

20. Centers for Disease Control and Prevention. Questions and Answers Regarding Creutzfeldt-Jakob Disease Infection Control Practices. Atlanta, GA: Centers for Disease Control and Prevention; 2000. Available at www.cdc.gov/ncidod/diseases/cjd/cjd_inf_ctrl_qa.htm.

21. Maheshwar A, De M, Browning ST. Reusable versus disposable instruments in tonsillectomy: a comparative study of outcomes. Int J Clin Pract 2003;57:579-583.

22. Nix P. Prions and disposable surgical instruments. Int J Clin Pract 2003;58:678-680.

23. U.S. Food and Drug Administration. Transcripts of the July 17 and 18, 2003 Transmissible Spongiform Encephalopathy Advisory Panel. Available at www.fda.gov/ohrms/dockets/ac/03/transcripts/3969t1.htm and www.fda.gov/ohrms/dockets/ac/03/transcripts/3969t2.htm.

24. Joint Commission on Accreditation of Healthcare Organizations. Sentinel Event Alert. Oakbrook Terrace, IL: Joint Commission on Accreditation of Healthcare Organizations; 2001. Available at www.jcaho.org/about+us/news+letters/sentinel+event+alert/print/ sea_20.htm.

25. Glatzel M, Abela E, Maissen M, Aguzzi A. Extraneural pathologic prion protein in sporadic Creutzfeldt-Jakob disease. $N$ Engl $J$ Med 2003;349:1812-1820.

26. Bruce ME, McConnell I, Will RG, Ironside JW. Detection of variant Creutzfeldt-Jakob disease infectivity in extraneural tissues. Lancet 2001;358:208-209.

27. Farling P, Popat M, Cooper S. Fiberoptic equipment and variant Creutzfeldt-Jakob disease. Anesthesia 2003;58:716-717.

28. Llewelyn CA, Hewitt PE, Knight RSG, et al. Possible transmission of variant Creutzfeldt-Jakob disease by blood transfusion. Lancet 2004;363:417-421.

29. Hill AF, Collinge I. Subclinical prion infection in humans and animals. British Medical Bulletin 2003;66:161-170. 